\title{
Cellulites Cervico-Faciales D'origine Dentaire au CHU Sylvanus Olympio de Lome Au Togo
}

\author{
Bissa Haréfétéguéna, $M D, M A$ \\ Adam Saliou, MD, MA \\ Amana Essobiziou, MD \\ Foma Winga, MD, MA \\ Pegbessou Essobozou, MD, MA
}

Université de Lomé, CHU Sylvanus Olympio, Lomé, Togo

Lawson Stephen Lionel, MD, MCA

Université de Kara, CHU Kara, Kara, Togo

Kamissoko Aly Badra, MD, CCA

Université Gamal Abdel Nasser,

Hôpital National Ignace Deen, Conakry, Guinée

Kpemissi Eyawèlohn, MD, Prof.

Université de Lomé, CHU Sylvanus Olympio, Lomé, Togo

\section{Doi:10.19044/esj.2019.v15n36p70～URL:http://dx.doi.org/10.19044/esj.2019.v15n36p70}

\section{Résumé}

Objectif : décrire les aspects épidémiologiques, cliniques, thérapeutiques, et évolutifs des cellulites cervico-faciales d'origine dentaire au CHU Sylvanus Olympio (SO) de Lomé. Patients et méthodes : Il s'est agi d'une étude transversale descriptive réalisée sur une période de sept ans, à propos de 373 dossiers médicaux colligés dans le service d'Oto-RhinoLaryngologie et de Chirurgie Cervico-Maxillo-Faciale du CHU SO de Lomé, comportant des informations cliniques et paracliniques faisant cas de cellulites cervico-faciales d'origine dentaire. Résultats : L'âge moyen de nos patients était de 38,5 ans $\pm 15,2$. Le délai moyen de consultation était de 11 jours. Les motifs les plus fréquents étaient la tuméfaction douloureuse $(94,1 \%)$ et l'odontalgie $(90,6 \%)$. Une automédication à base AINS/antalgiques a été retrouvée dans $33,0 \%$ des cas. Cliniquement, la tuméfaction était submandibulaire dans $60,1 \%$ des cas et associée à la carie dentaire dans $74,8 \%$ des cas. Les dents cariées étaient majoritairement les molaires inférieures dans $81,9 \%$ des cas. L'examen cytobactériologie des prélèvements a été réalisé chez 189 patients et 97 prélèvements se sont révélés positifs et isolant le streptocoque dans 32 cas. La radiographie panoramique a permis de noter un granulome dans $51,0 \%$ des cas, suivi du kyste apical dans $35,5 \%$ des cas. Le 
traitement médical était fait d'une bi-antibiothérapie réadaptée secondairement sur la base d'antibiogramme. Un drainage chirurgical avec extraction dentaire différée a été effectué dans $86,1 \%$ des cas. L’évolution était favorable chez la majorité des patients avec 6,9\% de décès. Conclusion : Les cellulites cervico-faciales d'origine dentaire restent d'actualité. Le diagnostic doit être précoce, la prise en charge est pluridisciplinaire mais un accent doit être mis sur la prévention.

Mots clés : Cellulite, Carie Dentaire, Automédication, Drainage Chirurgical

\title{
Head and Neck Cellulitis of Dental Origin at Sylvanus Olympio University Hospital of Lome in Togo
}

\author{
Bissa Haréfétéguéna, $M D, M A$ \\ Adam Saliou, MD, MA \\ Amana Essobiziou, $M D$ \\ Foma Winga, MD, MA \\ Pegbessou Essobozou, MD, MA
}

Université de Lomé, CHU Sylvanus Olympio, Lomé, Togo

Lawson Stephen Lionel, MD, MCA

Université de Kara, CHU Kara, Kara, Togo

Kamissoko Aly Badra, MD, CCA

Université Gamal Abdel Nasser,

Hôpital National Ignace Deen, Conakry, Guinée

Kpemissi Eyawèlohn, MD, Prof.

Université de Lomé, CHU Sylvanus Olympio, Lomé, Togo

\begin{abstract}
Objective: to describe the epidemiological, clinical, therapeutic and progressive aspects of head and neck cellulitis of dental origin at Sylvanus Olympio University Hospital (SO), Lomé. Material and method: This was a descriptive cross-sectional study carried out over a period of seven years, concerning 373 medical files collected in the Department of Oto-RhinoLaryngology and Head and Neck Surgery of the Sylvanus Olympio University Hospital in Lomé, with clinical and paraclinical information on head and neck cellulitis of dental origin. Results: The mean age of our patients was $38.5 \pm$ 15.2 years. The average consultation time was 11 days. The most common
\end{abstract}


reasons were painful swelling (94.1\%) and toothache (90.6\%). Selfmedication based on NSAIDs / analgesics was found in $33.0 \%$ of cases. Clinically, swelling was sub-mandibular in $60.1 \%$ of cases and associated with tooth decay in $74.8 \%$ of cases. The decayed teeth were predominantly lower molars in $81.9 \%$ of cases. The cytobacteriological examination of the specimens was carried out in 189 patients and 97 samples were positive and isolating the streptococcus in 32 cases. Panoramic X-ray revealed granuloma in $51.0 \%$ of cases, followed by apical cyst in $35.5 \%$ of cases. The medical treatment was made of a bi-antibiotherapy rehabilitated secondarily on the basis of antibiogram. Surgical drainage with delayed tooth extraction was performed in $86.1 \%$ of cases. The evolution was favorable for the majority of patients with $6.9 \%$ of deaths. Conclusion: Head and neck cellulitis of dental origin remains valid. The diagnosis must be early, the management is multidisciplinary but an emphasis must be placed on prevention.

Keywords: Cellulitis, Tooth Decay, Self-Medication, Surgical Drainage

\section{Introduction}

Les cellulites cervico-faciales sont des infections poly-microbiennes extensives, qui touchent les espaces aponévrotiques de la face et du cou. Souvent secondaires à la mortification de la pulpe dentaire ou aux infections péri-dentaires, elles se propagent par contiguïté et sont sévères parfois nécrosantes, susceptibles de s'étendre aux tissus avoisinants jusqu'au médiastin (Huang TT, 2004) constituant ainsi une urgence médico chirurgicale. Sa prévalence est encore élevée dans les pays en développement surtout en Afrique subsaharienne (Niang P et al., 2011). La prise en charge doit être rapide et pluridisplinaire. Le CHU Sylvanus Olympio de Lomé étant le seul centre de référence national qui abrite les chirurgiens maxillo-faciaux et la majorité des chirurgiens ORL, il s'avère judicieux d'y mener cette étude qui donne des renseignements complémentaires à celle menée antérieurement il y a 5 ans (Bissa $\mathrm{H}$ et al., 2014).

\section{Materiels Et Methode}

Le service d'Oto-Rhino-Laryngologie et de Chirurgie CervicoMaxillo-Faciale du Centre Hospitalier Universitaire Sylvanus Olympio (CHU-SO) de Lomé a servi de cadre d'étude. Il s'est agi d'une étude rétrospective descriptive de 07 ans qui s'est déroulée du $1^{\text {er }}$ janvier 2010 au 31 décembre 2016 et qui a porté sur 373 dossiers des patients hospitalisés pour cellulites cervico-faciales. Tous les cas ont rempli les critères de diagnostic suivants : informations cliniques et/ou paracliniques en faveur d'une cellulite cervico-faciale. N'ont pas été inclus dans cette étude les cellulites cervicofaciales d'origine non dentaire, les autres suppurations cervico-faciales et les 
dossiers incomplets. Les paramètres étudiés étaient les données sociodémographiques (âge, sexe, profession); cliniques (motif de consultation, antécédents, délai de consultation, signes cliniques) ; paracliniques (résultats biologique et radiologique); le traitement reçu et l'évolution. L'analyse et le traitement des données ont été faits avec le logiciel EPI info 7 et SPSS 20.0

\section{Resultats}

Au total, 373 cas ont rempli les critères d'inclusion, ce qui représente 53,3 cas par an. L'âge moyen était de 38,5 ans $\pm 15,2$ avec des extrêmes de 3 et 85 ans. La tranche d'âge la plus représentée était celle de 30-45 ans soit $33,7 \%$ des cas. (Figure 1). Cent quatre-vingt-seize patients étaient de sexe masculin soit une sex-ratio de 1,1 .

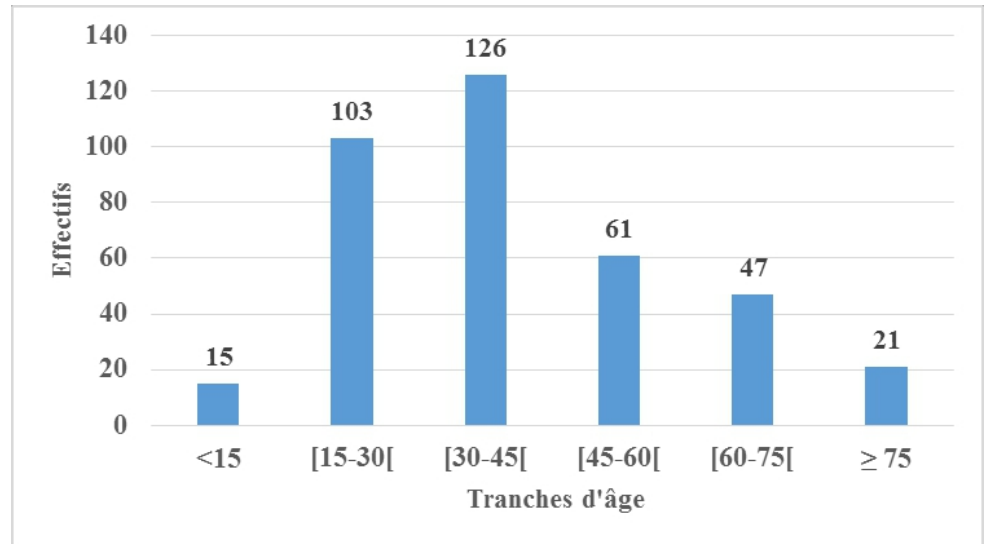

Figure 1: répartition des patients selon les tranches d'âge

Les commerçants et les ménagères ont représenté respectivement $24,4 \%$ et $14,7 \%$ des cas. (Tableau I). Le délai moyen de consultation était de 11 jours avec des extrêmes de 1 et 42 jours et 191 cas $(51,2 \%)$ avaient consulté dans les sept premiers jours d'évolution de la maladie. La tuméfaction douloureuse et l'odontalgie étaient les motifs de consultation les plus retrouvés soit dans respectivement $94,1 \%$ et $90,6 \%$ des cas, suivi de dysphagie dans $14,5 \%$ des cas, de dyspnée dans 3,8\% des cas, d'un mauvais état général dans $1,9 \%$ des cas et des suppurations cervicale, buccale et faciale dans respectivement $0,8 \% ; 0,8 \%$ et $0,5 \%$ des cas. L'automédication était retrouvée dans 212 cas $(56,8 \%)$ et faite d'association anti-inflammatoires non stéroïdiens et antalgiques dans $33,0 \%$ des cas , d'antalgiques uniquement dans $29,2 \%$ des cas , d'anti-inflammatoires non stéroïdiens uniquement dans $23,5 \%$ des cas et des antibiotiques uniquement dans $14,1 \%$ des cas. 
Tableau I: répartition des patients en fonction de la catégorie professionnelle

\begin{tabular}{lcc}
\hline Catégorie professionnelle & Effectifs & Pourcentage(\%) \\
\hline Artisans & 46 & 12,3 \\
Agriculteurs & 27 & 7,2 \\
Cadres supérieurs & 11 & 2,9 \\
Commerçants & 91 & 24,4 \\
Conducteurs de taxi-moto & 15 & 4,0 \\
Elèves/Etudiants & 38 & 10,3 \\
Ménagères & 55 & 14,8 \\
Ouvriers/Employés & 43 & 11,5 \\
Retraités & 25 & 6,7 \\
Autres & 22 & 5,9 \\
Total & $\mathbf{3 7 3}$ & $\mathbf{1 0 0 , 0}$ \\
\hline
\end{tabular}

Quant aux antécédents, l'alcoolisme chronique était retrouvé dans $8,31 \%$ des cas, l'immunodépression au VIH dans $6,97 \%$ des cas, l'extraction dentaire dans $4,83 \%$ des cas, le tabagisme chronique dans $3,48 \%$ des cas, le diabète dans $2,41 \%$ des cas, la cellulite cervico-faciale et une mauvaise hygiène buccodentaire dans $1,07 \%$ des cas chacune et un traumatisme dentaire dans $0,8 \%$ des cas.

L'examen physique exo-buccal a permis de noter une tuméfaction siégeant dans la région submandibulaire dans $60,1 \%$ des cas. (Tableau II). (Figure 2). L'examen endo-buccal a permis de noter un trismus dans 67,3\% des cas et qui était modéré dans $63,2 \%$ des cas, serré dans $29,1 \%$ des cas et léger dans $7,7 \%$ cas ; des caries dentaires étaient retrouvées dans 279 cas soit $74,8 \%$ des cas dont une dent cariée dans $48,38 \%$ des cas, deux dents dans $28,67 \%$ des cas, trois dents dans $12,18 \%$ des cas, quatre dents dans $7,17 \%$ des cas et supérieure à quatre dents dans 3,6\% des cas. Quant au type de dent cariée, les molaires inférieures étaient retrouvées dans $81,3 \%$ des cas, les molaires supérieures dans 2,9\% des cas, les prémolaires inférieures dans 9,7\% des cas, les prémolaires supérieures dans $1,8 \%$ des cas et les autres dents dans $4,3 \%$ des cas. Les autres signes retrouvés à l'examen endo-buccal étaient une absence de dent dans $20,4 \%$ des cas, des fistules dans $19,3 \%$ des cas, un aspect en double langue dans $13,7 \%$ des cas et une parodontopathie dans $13,1 \%$ des cas.

Tableau II : répartition des patients selon la localisation de la tuméfaction

\begin{tabular}{lcc}
\hline Localisation de la tuméfaction & Effectifs & Pourcentage(\%) \\
\hline Cervico-facio-thoracique & 38 & 10,1 \\
Génienne & 37 & 10,0 \\
Hémiface & 34 & 9,1 \\
Parotido-massétérienne & 14 & 3,7 \\
Périorbitaire & 1 & 0,3
\end{tabular}




\begin{tabular}{lcc} 
Plancher buccal sus mylo hyoïdienne & 23 & 6,2 \\
Submandibulaire & $\mathbf{2 2 4}$ & $\mathbf{6 0 , 1}$ \\
Temporale & 2 & 0,5 \\
Total & $\mathbf{3 7 3}$ & $\mathbf{1 0 0 , 0}$ \\
\hline
\end{tabular}

A

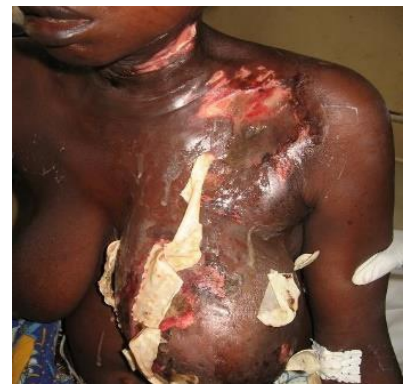

B

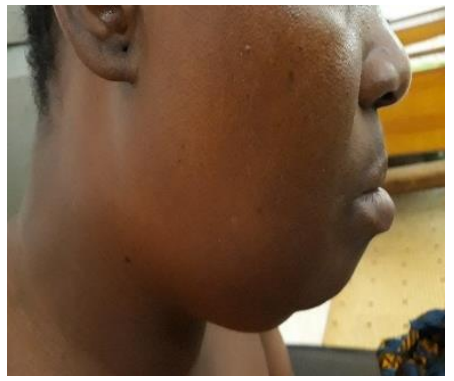

Figure 2: $\mathbf{A}$ = cellulite cervico-facio-thoracique ayant entrainé une médiastinite $\mathbf{B}=$ cellulite submandibulaire droite.

L'examen cytobactériologique du pus a été réalisé dans 189 cas $(50,73 \%)$ et des germes étaient isolés dans 97 cas $(51,3 \%)$. Les germes isolés étaient le streptocoque dans $32,9 \%$ des cas, le staphylocoque dans $22,6 \%$ des cas, le klebssiela dans $18,55 \%$ des cas, l'Escherichia coli dans $11,34 \%$ des cas, le Pseudomonas aueroginosa dans $8,25 \%$ des cas et le citrobacter divertus, l'anterobacter, le Proteus vulgaris dans $6,36 \%$ des cas.

La radiographie panoramique dentaire était réalisée dans 149 cas $(39,94 \%)$. (Figure 3) Elle avait noté au niveau apical des granulomes dans $51,0 \%$ des cas et des kystes dans $35,5 \%$ des cas ; des dents de sagesse incluse et enclavée dans respectivement $6,7 \%$ et $6,0 \%$ des cas, et une ostéite dans $0,6 \%$ des cas.

A

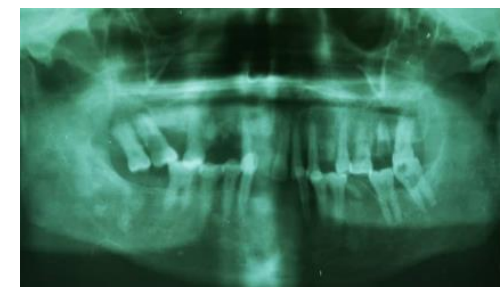

B

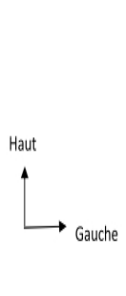

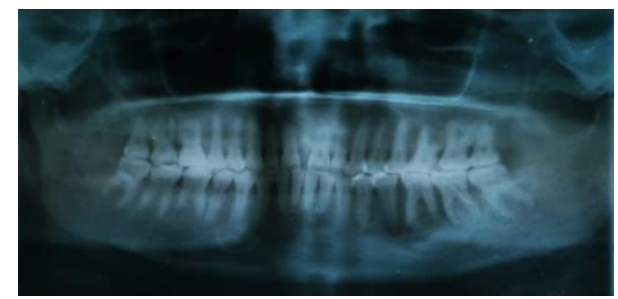

Figure 3 : radiographie panoramique dentaire, $\mathbf{A}=$ multiples caries et chicots $/ \mathbf{B}=$ granulome apical (flèche)

Le traitement était fait de traitement médical et chirurgical. Le traitement médical était une bi antibiothérapie probabiliste dans tous les cas puis réadapté en fonction du germe et de l'antibiogramme dans 97 cas. Le drainage exo- 
buccal suivi d'une extraction dentaire différée ont été réalisés dans $86,1 \%$ des cas (Tableau III).

Tableau III : répartition des patients en fonction du type de geste chirurgical

\begin{tabular}{lcc}
\hline Geste chirurgical & Effectifs & Pourcentage(\%) \\
\hline Endo-buccal + extraction dentaire différée & 2 & 0,5 \\
Exo-buccal + extraction dentaire différée & $\mathbf{3 2 1}$ & $\mathbf{8 6 , 1}$ \\
Exo-buccal + extraction dentaire à chaud & 6 & 1,6 \\
Pas de traitement chirurgical & 44 & 11,8 \\
Total & $\mathbf{3 7 3}$ & $\mathbf{1 0 0 , 0}$ \\
\hline
\end{tabular}

La durée moyenne de séjour hospitalier était de 11,3 jours $(57,6 \%)$ avec des extrêmes de 07 jours et 21 jours. L'évolution était simple et favorable dans 270 cas soit $72,4 \%$ des cas avec 26 cas $(6,9 \%)$ de décès. Des complications ont été notées dans 7 cas $(1,8 \%)$ dont 1 cas d'extension orbitaire, 1 cas d'insuffisance rénale, 2 cas de médiastinite, et 3 cas de pleurésie (Figure 4).

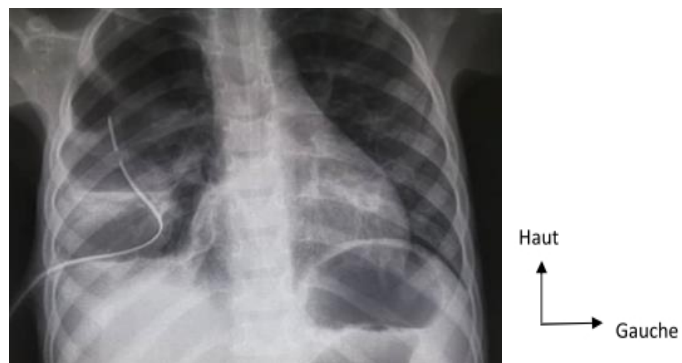

Figure 4 : pleurésie basale droite avec mise en place d'un drain thoracique (flèche)

\section{Discussion}

Les cellulites cervico-faciales constituent le premier motif d'hospitalisation dans le milieu où exercent les auteurs. La prévalence dans cet article était de $16,5 \%$, elle varie en fonction du milieu où l'étude a été réalisée (Rouadi $\mathrm{S}$ et al., 2013). Le sexe masculin était le plus retrouvé et ce résultat fait l'unanimité des auteurs (El Abed W et al. 2019; Tran ba huy et al., 2011 ; Benzarti S et al., 2007 et Rakotoarison RA et al., 2008) ; les causes évoquées sont essentiellement une mauvaise hygiène bucco-dentaire et l'alcoolo tabagisme rencontrée plus chez le sexe masculin (El Ayoubi A et al., 2009). Les cellulites cervico-faciales surviennent à tout âge. L'âge moyen des patients retrouvé ici était de 38,5 ans avec un pic dans la tranche d'âge de 30-45 ans. Dans certaines études, cet âge moyen variait de 28 à 38 ans (Miloundja et al., 2012 et Cassagneau P et al., 2011), une étude béninoise 
(Hounpke et al ., 1990) avait noté un âge moyen de 18 ans et pour Potard et al. en France, cet âge était de 51 ans (Potard G et al., 2000).

Les commerçants et les ménagères représentaient les classes professionnelles les plus touchées dans cet article. Ce profil a été noté dans certaines études africaines comme dans celle de Bissa et al. au Togo qui ont noté 87,9 \% (Bissa $\mathrm{H}$ et al., 2014) et une autre en Tunisie ( El Abed W et al. 2019) qui a noté $71,8 \%$ des patients à bas niveau socio-économique. Cette catégorie professionnelle accorderait peu d'importance à l'hygiène buccodentaire, plus d'importance à l'automédication qui n'a jamais été efficace dans la majorité des cas. Elles consulteront alors tardivement devant l'aggravation de la maladie.

Le retard de consultation est dû à la paupérisation, à la résignation, à l'automédication et aussi au traitement traditionnel. Ces causes ont été rapportées par certaines études Sénégalaise et gabonaise (Niang $\mathrm{P}$ et al., 2011 et Miloundja et al., 2012) . Le délai moyen de consultation retrouvé dans cet article était de 11 jours. Par contre un délai moyen plus court soit 4 jours a été noté par certains auteurs en Tunisie (Benzarti S et al., 2007 et El Abed W et al., 2019). Ceci pourrait s'expliquer par la meilleure organisation du système de santé de ces pays maghrébins.

La tuméfaction douloureuse $(94,1 \%)$ était le motif de consultation le plus retrouvé dans cet article et souvent associé à une odontalgie (90,6\%). c'est le motif qui fait suspecté une cellulite cervico-faciale et reste généralement au premier plan (El Ayoubi A et al., 2009 et Hounpke et al ., 1990) . Cette tuméfaction siégeait plus dans la région mandibulaire dans $60,1 \%$ des cas. Cette localisation a été rapportée dans la littérature (Benzarti S et al., 2007 et Rouadi S et al., 2013). La raison de cette localisation est liée à la cause qu'est la carie dentaire qui touche principalement les dents mandibulaires, siège préférentiel de la formation de la plaque dentaire (El Abed W et al., 2019 et El Ayoubi A et al., 2009).

Le recours à l'automédication a été noté dans plus de la moitié des cas. L'automédication est une pratique courante dans les pays en voie de développement à cause de la vente des médicaments de la rue, de la médecine traditionnelle et de l'absence de couverture des assurances maladies aux autres couches sociales. Des auteurs africains l'ont aussi noté dans leurs études (E1 Ayoubi A et al., 2009 et Miloundja et al., 2012). Par contre une étude française rapporte moins de patients qui ont eu recours à l'automédication soit 8,6\% des cas (Tran ba huy et al., 2011). En effet, en France et dans les pays où le système de santé est modernisé, la délivrance d'un médicament par le pharmacien se fait sur présentation d'une ordonnance médicale, d'où un faible taux d'automédication.

La majorité des études sont unanimes sur la nature des germes rencontrés à l'examen cytobactériologique du pus. Ce sont des germes 
saprophytes et commensaux aéro- anaérobies de la cavité buccale, localisés souvent dans les sillons dentaires (El Abed W et al. 2019 ; Benzarti S et al., 2007 et El Ayoubi A et al., 2009). Comme démontré ici, 97 prélèvements $(51,3 \%)$ étaient positifs et ont isolé les germes dans les proportions suivantes : le streptocoque $(32,9 \%)$, klebsiella (19\%), staphylocoque (22,6\%). Le streptocoque est très souvent le germe causal de la cellulite d'origine dentaire (Miloundja et al., 2012). Des études antérieures effectuées dans le même service, avaient retrouvé des résultats similaires (Bissa $\mathrm{H}$ et al., 2014 et Kpémissi E , 1995).

Le bilan radiologique est indispensable dans le diagnostic et la surveillance des cellulites cervico-faciales. La radiographie panoramique dentaire et la TDM thoraco cervicale s'imposent donc de façon systématique. La radiographie panoramique dentaire a permis d'objectiver dans cet article des lésions dentaires diverses parmi lesquelles les kystes et les granulomes apicaux. Les autres types de lésions retrouvées dans la littérature sont les chicots, les kystes radiculo-dentaires, les foyers d'alvéolites et parfois les tumeurs et pseudo tumeurs sous-jacentes (El Ayoubi A et al., 2009). Pour mieux explorer l'extension de la cellulite surtout la recherche d'une collection profonde et de diagnostiquer une médiastinite ou une thrombophlébite septique, une TDM cervico-thoracique avec injection s'avère indispensable (Cassagneau P et al., 2011).

L'antibiothérapie a révolutionné le pronostic des cellulites cervicofaciales d'origine dentaire. Elle permet de traiter le germe en cause et de ralentir l'extension de l'infection. Elle est nécessaire mais non suffisante en cas de collections suppurées ou de plages de nécrose (Bennani-Baïti AA, 2015). Les antibiotiques utilisés dans les cellulites sont souvent les bétalactamines, les dérivés imidazolés, les aminosides et les macrolides (Dubreuil L et al., 2005). Le drainage chirurgical a une grande valeur thérapeutique devant une cellulite collectée. Le drainage exo-buccal suivi d'une extraction dentaire différée a été l'attitude thérapeutique la plus pratiquée dans cet article. Dans certaines études (Rakotoarison RA et al., 2008), les mêmes gestes ont été effectués. A cause du trismus et de l'état infectieux, il est préférable de pratiquer l'extraction dentaire de façon différée (El Abed W et al. 2019, et El Ayoubi A et al.,2009). En présence de signes de compressions ou de diffusion thoracique, une trachéotomie ou une thoracotomie peut être pratiquée en complément du drainage cervico-facial (Rouadi S et al., 2013 et El Abed W et al., 2019).

Le taux de mortalité varie entre 0 et $50 \%$ dans la littérature (Benzarti S et al., 2007 et Robertson D et al., 2009). Comme démontré ici ; 6,9\% de décès était dû au choc septique secondaire à une diffusion thoracique. Lorsque la consultation et la prise en charge sont précoces et efficaces, il est possible de préserver le pronostic vital. Pour Rouadi et al., une hospitalisation 
d'emblée en réanimation des cas graves pourrait réduire le taux de mortalité (Rouadi S et al., 2013). Dans cet article, le retard de consultation expliquerait cette fréquence de décès.

\section{Conclusion}

Les cellulites cervico-faciales sont des affections graves touchant plus les adultes jeunes. Les motifs de consultation fréquemment retrouvés étaient la tuméfaction douloureuse et l'odontalgie. L'examen clinique a permis de noter une tuméfaction douloureuse siégeant en région submandibulaire associée aux caries dentaires. Les dents cariées étaient majoritairement les molaires inférieures. L'examen cytobactériologique et la radiographie panoramique sont les examens demandés à visée étiologique qui ont permis respectivement d'isoler les germes et d'objectiver des lésions dentaires. En comparaison de l'étude menée précédemment dans le même service, il ressort que la flore bactérienne demeure identique. Le traitement médico chirurgical était basé sur une antibiothérapie associée à un drainage chirurgical avec extraction dentaire différée. L'évolution était favorable dans la majorité des cas.

\section{References:}

1. Bennani-Baïti AA. (2015). Ann Otolaryngol Chir Cervicofac; $132: 169-73$.

2. Benzarti S, Mardassi A, Mhamed R, Hachicha A, Brahem H, Akkari K, Miled I, Chebbi K. (2007). Les cellulites cervico-faciales d'origine dentaire: à propos de 150 cas. j tun orl ; 19(1) :24-7.

3. Bissa H, Salou M, Pégbéssou EP, Amana B, Dossim S, Tigossou S, Dagnra AY, Kpemissi E. (2014). Aspects épidémiologiques et bactériologiques des cellulites cervico-faciales au CHU Sylvanus Olympio de Lomé. Rev Af d'ORL et de chir cervico-fac ; 14 :32-6

4. Cassagneau P, Varoquaux A, Moulin G. (2011). Exploration radiologique des infections cervico-faciales. J RADIOL ; 92 :1015-28

5. Dubreuil L, Neut C. (2005). Arguments microbiologiques pour optimiser l'antibiothérapie empirique des cellulites cervico-faciales. Med Buc Chir Buc ; 11 :7-15

6. El Abed W, Krimi H, Lezid S, Khribi M, Gnaba K. (2019). Facteurs prédictifs de gravité des cellulites cervico-faciales:a propos de 180 observations. j. tun orl; $41: 41-5$

7. El Ayoubi A, El Ayoubi F, Mas E, Guertite A, Boulaïch M, Essakalli L, Benbouzid A, Kzadri M. (2009). Cellulites cervico-faciales diffuses d'origine dentaire: à propos de 60 cas. Med Buc Chir Buc ; 15(3):12735 . 
8. Hounkpe YYC, Oussa GB, Vodouhe SJ, Babagbeto MJ, Medji ALP, Bassabi SK. (1990). Les cellulites cervico-faciales: à propos de 55 cas colligés dans les services d'ORL et d'Ophtalmologie du CNHU de Cotonou. Med Afr Noire ; 37(1):29-34.

9. Huang TT, Liu TC. (2004). Deep neck infection: analysis of 185 cases. Head Neck ; 26 (10):854-60.

10. Kpémissi E. (1995). Cellulites cervico-faciales d'origine buccodentaire: étude de 26 cas au CHU de Lomé. Rev Laryngol Otol Rhinol;116(3):195-7.

11. Miloundja J, Eyogho SFA, Lawson JMM, Ondounda M, Koumba JS, Lekassa P, Inibend M, N'zouba L. (2012). Cellulites cervico-faciales diffuses: 32 cas vus à Libreville. Cah Etu Rech Fran/ Santé ; 21(3):1537.

12. Niang P, Ba A, Tamba-Fall A, Dia Tine S, Kounta A, Diop R, Tamba B, Bouassalo KM, Gassama-Barry CB, Toure S, Diallo B. (2011).Aspects epidemiologiques et cliniques des cellulites perimaxillaires : experience d'un service de stomatologie au senegal Rev. Col. Odonto-Stomatol. Afr. Chir. Maxillo-fac ; 18(3) : 33-8

13. Potard G, Marianowski R, Fortun C, Raybaud O, Preveraud D, Vazel L, Martins C, Jezequel JA. (2000).Cellulites de la face et du cou: à propos de 13 cas. J Fran oto-rhino-laryngol;49(6):325-37

14. Rakotoarison RA, Ramarozatovo NP, Rakoto FA, Rakotovao FJ. (2008). Cellulites cervico-faciales: à propos de 41 cas. Med Buc Chir Buc ; 14(1):35-9

15. . Robertson D and Smith AJ. (2009).The microbiology of the acute dental abscess. J Med Microbiol ; 58: 155-62

16. Rouadi S, Ouaissi L, El Khiati R, Abada R, Mahtar M, Roubal M, Janah A, Essaadi M, Kadiri F. (2013). Les cellulites cervico-faciales à propos de 130 cas. PAMJ ;14(1) : 88-93.

17. Tran Ba Huy P, Blancal JP, Verillaud B. (2011). Les cellulites cervicofaciales : Une grave urgence ORL. Bull. Acad. Natle. Med;195(3):66178. 